\title{
The Zero-Condition: A Simplifying Assumption in QALY Measurement and Multiattribute Utility
}

\author{
John M. Miyamoto • Peter P. Wakker • Han Bleichrodt • Hans J. M. Peters \\ Department of Psychology, University of Washington, Seattle, Washington 98195 \\ Department of Medical Decision Making, LUMC 1 K6-R, Leiden University Medical Center, \\ P.O. Box 9600, Leiden 2300 RC, The Netherlands \\ iMTA, Erasmus University, P.O. Box 1738, Rotterdam, 3000 DR, The Netherlands \\ Department of Quantitative Economics, Maastricht University, P.O. Box 616, Maastricht, 6200 MD, The Netherlands
}

$\mathrm{T}$ his paper studies the implications of the "zero-condition" for multiattribute utility theory. The zero-condition simplifies the measurement and derivation of the Quality Adjusted Life Year (QALY) measure commonly used in medical decision analysis. For general multiattribute utility theory, no simple condition has heretofore been found to characterize multiplicatively decomposable forms. When the zero-condition is satisfied, however, such a simple condition, "standard gamble invariance," becomes available. (QALY; Multiattribute Utility; Utility Independence)

\section{Introduction}

Quality adjusted life years (QALYs) provide an important and widely applied outcome measure in medical decision analysis. They constitute a well-founded method for combining quality of life and life duration into a single measure that serves as a criterion for tradeoffs in economic analyses. Their meaning and interpretation is clear, and they are reasonably tractable. There are, however, some methodological problems. QALYs are based on a number of assumptions about utility and individual preference that are, at best, satisfied to some degree of approximation (Loomes and McKenzie 1989). For a precise assessment of the usefulness of QALYs, a deeper insight into their intrinsic meaning is warranted, and that is the subject of this paper.

The tractability of QALY calculations is based on three simplifying assumptions. First, utility of life duration is assumed to be linear. Second, a multiplicative combination of life duration and health is assumed. Third, for nonchronic health states, values of different periods with different health states are added up. This last assumption may be the most ques-

0025-1909/98/4406/0839\$05.00

Copyright (c) 1998, Institute for Operations Research

and the Management Sciences tionable one, but is not the subject of this paper (Johannesson 1995). Hence attention is restricted throughout this paper to chronic health states. The assumption of linear utility for duration is sometimes weakened to permit discounting or risk aversion. We consider alternative derivations of the linear and nonlinear cases of the QALY model.

It is not always recognized that a multiplicative combination is immediately suggested by some obvious characteristics of health preferences. First, some health states are worse than death. Whereas most health states are "positive" (longer life durations are preferred to shorter ones), worse-than-death states are ones in which the opposite is true. This inversion of preferences suggests a multiplicative rule because multiplication by a negative number produces an analogous inversion of inequalities. A second phenomenon that reflects the idea of multiplication concerns the "empty" life duration of 0 years. When survival duration is 0 years (the vacuous time period), it obviously does not matter what the state of health is. This phenomenon also suggests a multiplicative rule because multiplication by 0 nullifies 
all differences. Below we formally define the second phenomenon as the "zero-condition" for preference and we study its implications.

This paper provides a derivation of QALYs that reflects as closely as possible the empirical phenomena just described. Thus we obtain a characterization of the meaning of QALYs that is more fundamental than those provided hitherto in the literature. The characterization reveals more plainly the critical issues in the discussions for and against the use of QALYs. Our derivation will also be more elementary and easier to understand than the previous derivations. Not only do we drop the commonly used assumption of constant proportional tradeoffs, but also we drop the complicated assumptions of utility independence (in the case of linear utility) or we replace the utility independence assumption by a weaker assumption, standard gamble invariance (in the case of nonlinear utility for life duration). These simplifications are achieved through the logical power of the zero-condition. It is our deliberate aim that the analysis of this paper be as elementary as possible in order to make the intrinsic meaning of QALYs as transparent as possible.

The measurement of QALYs belongs to the general domain of multiattribute utility (Keeney and Raiffa 1976). The QALY is a two-attribute multiplicative utility function. Section 5 studies implications of the zero-condition for general multiattribute utility theory where there can be more than two attributes. Theorem 5.1 provides a general characterization, for two or more attributes, of multiplicative representations when signs can change; it simplifies existing results. Versions of the mathematical zero-condition have appeared in several studies in the past. We discuss these, and show how our theorem generalizes the existing results.

Section 2 presents our analysis of QALYs with linear utility for life duration. The zero-condition is introduced and discussed. The third section extends the analysis to nonlinear utility, allowing for general risk attitudes and discounting. Section 4 compares our results to existing results on QALY measurement in the literature and presents examples and further discussion. The next section considers general multiattribute utility theory. We conclude with a brief discussion of the elementary nature of our analysis. Formal proofs are given in an appendix.

\section{QALYs under Risk Neutrality}

A pair $(Q, T)$ designates an outcome where a person lives for $T$ years in health state $Q$ and then dies; i.e., outcomes in this paper are chronic health states. A gamble is a probability distribution over chronic health states, in other words, a gamble specifies the probabilities with which different chronic states occur. We assume that preferences over gambles satisfy expected utility theory as derived by von Neumann and Morgenstern (1944) and denote the utility function by $U$ (Weinstein et al. 1980, Sox et al. 1986, Clemen 1991). The standard QALY model assumes risk neutrality with respect to life duration, meaning that an uncertain lifetime of expected duration $T$ is equally preferred to a certain lifetime of duration $T$ when health quality is held fixed. This assumption underlies the time tradeoff method (defined in \$4) which is widely used to measure health quality, and it is used in Markov analyses of nonchronic health states. The present section considers the standard QALY model; the next section generalizes the discussion to QALYs with risk aversion.

The QALY model asserts that $Q$ and $T$ are related as follows:

$$
U(Q, T)=V(Q) \cdot T .
$$

In other words, the utility of duration is linear and $V(Q)$ represents a correction factor for health quality. Figure 1 illustrates the QALY model for a few health states.

\section{Figure 1 QALY Utility Functions for Life Duration, for Five Health States}

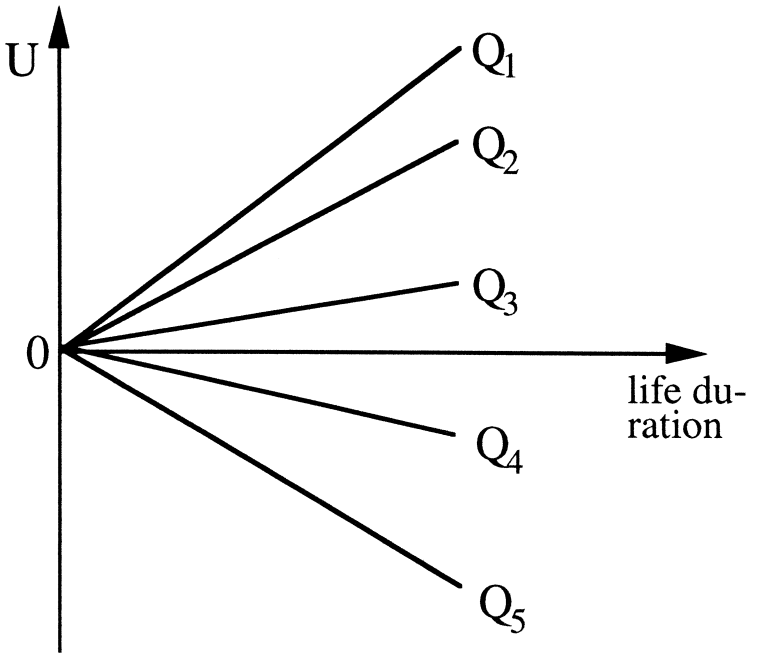

MAnAgement Science/Vol. 44, No. 6, June 1998 
Two critical features of the QALY model are evident in Figure 1. First, the utility of life duration is linear, implying risk neutrality. Second, the multiplicative relationship between $Q$ and $T$ implies that all utility lines radiate from the origin. Figure 1 also depicts worsethan-death health states; their utility lines descend from the origin. The focus of this paper is to highlight the next preference assumption whose importance for utility modeling has not been widely recognized.

Preferences satisfy the zero-condition if all health states are equally preferred when the duration is 0 . The zerocondition means that $(Q, 0)$ is equivalent in preference to $\left(Q^{\prime}, 0\right)$ for all health states $Q, Q^{\prime}$. Later we will present a more complex set of assumptions from which the QALY model is commonly derived in the literature. Here we point out a rather surprising result-the QALY model is already implied by risk neutrality and the zerocondition.

THEOREM 2.1. Under expected utility, the QALY model holds if and only if risk neutrality and the zero-condition hold.

To illustrate the preference conditions, Figure $2 \mathrm{a}$ shows a possible utility structure in which risk neutrality is satisfied but the zero-condition is not. Obviously for each health state $Q$, the utility function for survival in $Q$ is linear, but the functions for different $Q$ can lie

Figure 2a Utility Functions for Life Duration, for Risk Neutrality but without the Zero-Condition

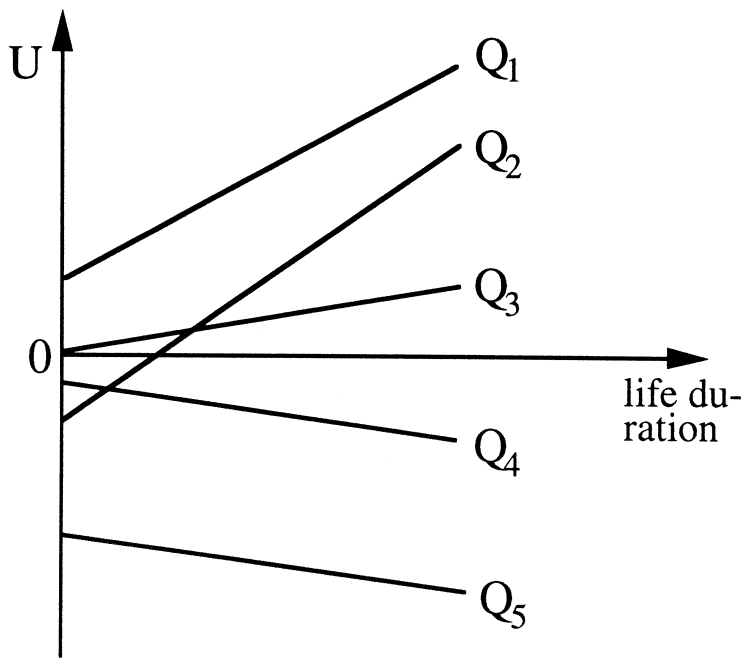

Figure 2b QALY Utility Functions for Life Duration, for the ZeroCondition but without Risk Neutrality

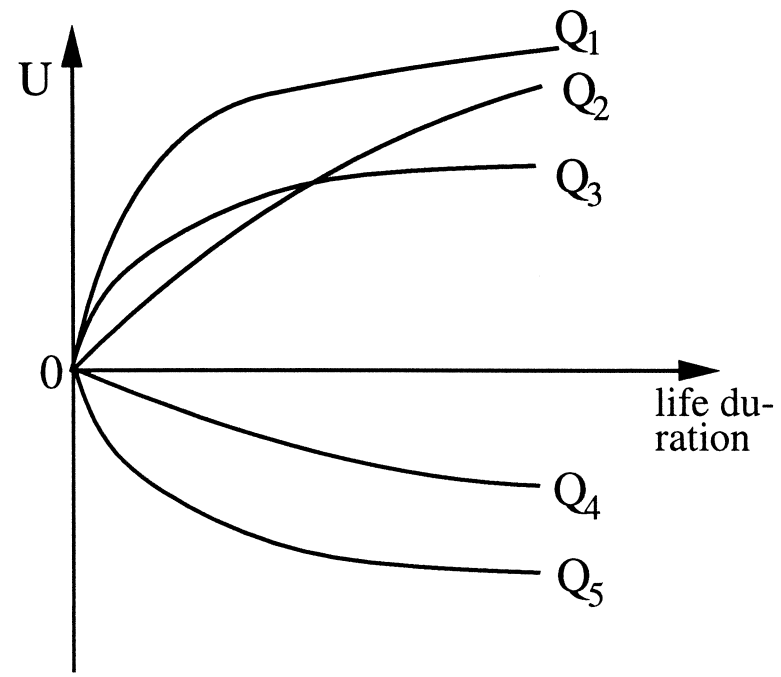

anywhere in the plane (Bleichrodt et al. 1997). The zerocondition is not assumed because the utility functions do not all coincide at $T=0$. For example, Figure 2a implies that duration 0 in health state $Q_{1}$ would be preferred to duration 0 in health state $Q_{2}$. Figure $2 \mathrm{~b}$ shows a possible utility structure in which the zero-condition is satisfied but risk neutrality is not. The utility functions can follow any nonlinear pattern, but they must coincide at $T=0$. It is convenient to locate the common value at the origin, and this has been done in Figure $2 b$. Clearly the combination of the constraints shown in Figures $2 \mathrm{a}$ and $2 \mathrm{~b}$ together yield a utility structure as in Figure 1. This reasoning captures the proof of Theorem 2.1.

A health state is positive if longer durations are preferred to shorter durations; in the QALY model this means that $V(Q)>0$. A health state is negative if shorter durations are preferred to longer durations, which means that $V(Q)<0$ in the QALY model. Sometimes it can be useful to incorporate health states $Q$ for which all life durations are equivalent in preference to immediate death. Survival in such health states is neither preferred to nor less preferred than death. Such health states will be called equal-to-death health states in this paper. Equal-to-death health states are represented in the QALY model by the valuation $V(Q)=0$. Health states that are not equal-to-death will be called unequal- 
to-death health states. Theorem 2.1 generalizes an earlier finding that was restricted to positive health states (Bleichrodt et al. 1997).

The status of the zero-condition is subtle and deserves further discussion. If it is, as we claim, completely evident, then how is it possible that this apparently vacuous condition can have so many implications? To understand this point, note that in formal mathematics there is no reason why an abstract pair (healthy, 0) would designate the same object as an abstract pair (ill, 0 ). These two objects are, however, identical physical objects in our domain of application where the second dimension designates duration of time. This identity is beyond empirical testing and is a logical implication of the physical meaning of pairs $(Q, T)$ in our domain. Therefore, there is no medical interest in mathematical models with differences between a pair (healthy, 0 ) and (ill, 0 ). In summary, violations of the zero-condition are impossible in the measurement of health outcomes.

\section{QALYs without Risk Neutrality}

The assumption of linear utility in the previous section was motivated by the tractability it provides for calculations; in particular, it permits the use of the time tradeoff technique for eliciting health state utilities. It has been used in theoretical studies by de Finetti (1937) and Nau (1992). The assumption is not empirically realistic, however, because it does not incorporate discounting and risk aversion. A more realistic measure results if we drop the assumption of linearity, leading to generalized QALYs:

$$
U(Q, T)=V(Q) \cdot W(T)
$$

where $W(0)=0$. Here $W$, the function that values life duration, can be nonlinear. Figure 3 is an example of a generalized QALY utility structure. Utility functions for life duration have the same curvature with respect to different health states, and they all coincide at $T=0$.

The justification of this model in terms of preference conditions becomes more complicated because risk neutrality can no longer be invoked here. In place of risk neutrality, we adopt a condition on standard gambles for life duration. Consider any health state $Q$ and a standard gamble equivalence where $(Q, T)$ is equal in preference to a $p$ chance of $(Q, Y)$ and a $1-p$ chance of $(Q$,
$Z)$. We call $p$ the probability equivalent of $(Q, T)$ with respect to the end points, $(Q, Y)$ and $(Q, Z)$. Under risk neutrality, $p$ equals $(T-Z) /(Y-Z)$, but it will be different under risk aversion or risk seeking. Therefore we relax the assumption that $p$ equals $(T-Z) /(Y-Z)$ in this section, but we keep the assumption that probability equivalents are the same in different unequal-todeath health states. That is, if $Q$ and $Q^{\prime}$ are unequal-todeath and $p$ is the probability equivalent of $(Q, T)$ with respect to $(Q, Y)$ and $(Q, Z)$, then $p$ is also the probability equivalent of $\left(Q^{\prime}, T\right)$ with respect to $\left(Q^{\prime}, Y\right)$ and $\left(Q^{\prime}, Z\right)$. This condition is called standard gamble invariance (SG invariance). The definition of SG invariance is restricted to unequal-to-death health states because every duration is equivalent to every gamble for survival in an equal-to-death health state.

A remarkable feature of the multiplicative model can be inferred from Figure 3. That is, if the commonly found risk aversion holds for positive health states, then risk seeking is implied for negative health states. This property, risk aversion for positive outcomes but risk seeking for negative outcomes, agrees well with the predictions of prospect theory (Fishburn and Kochenberger 1979, Currim and Sarin 1989, Tversky and Kahneman 1992).

The following theorem demonstrates that under SG invariance, the zero-condition has essentially the same force as it had in the previous section.

\section{Figure 3 QALY Utility Functions for Life Duration, for the Zero-Condition but without Risk Neutrality}

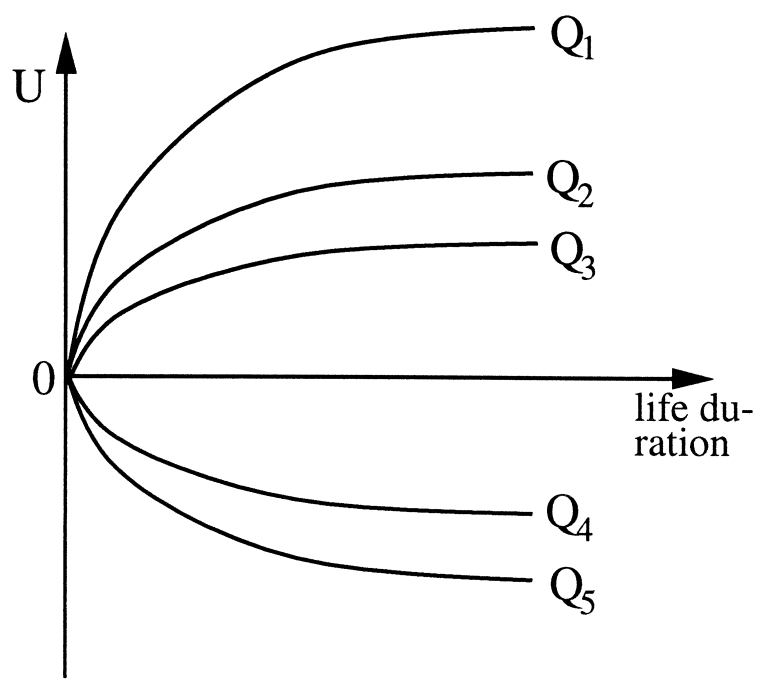

MAnAGEMEnt SCIEnCE/Vol. 44, No. 6, June 1998 
Figure 4 Utility Functions for Life Duration with SG Invariance but without the Zero-Condition

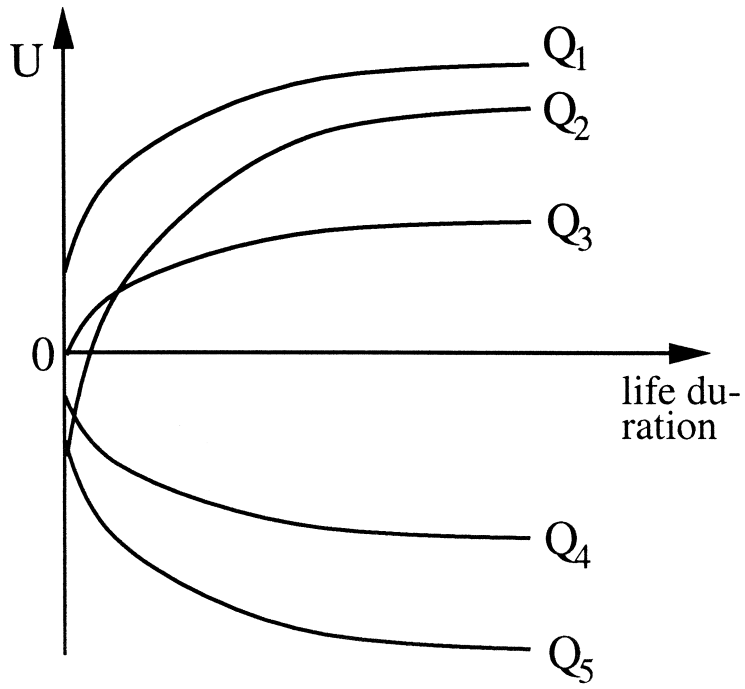

THEOREM 3.1. Under expected utility, the generalized QALY model holds if and only if the zero-condition and SG invariance hold.

Figure 4 shows a possible utility structure in which SG invariance is satisfied but the zero-condition is not. All of the utility functions have the same curvature (possibly inverted), but they have different slopes and intercepts. The zero-condition is not satisfied because the utility functions do not all coincide at $T=0$. If the constraint of SG invariance, depicted in Figure 4, is combined with the zero-condition as depicted in Figure 2b, then a utility structure as in Figure 3 results. This reasoning captures the proof of Theorem 3.1.

It is convenient to set $V(H)=1$ for perfect health $H$ and to assume that $W$ is increasing in life duration. $\mathrm{Ob}-$ viously in the generalized QALY model, positive health states are represented by the value $V(Q)>0$, negative health states are represented by the value $V(Q)<0$, and equal-to-death health states are represented by the value $V(Q)=0$.

\section{Comparison to Existing QALY Derivations and Discussion}

The assumptions that have been invoked in the literature to derive QALYs and generalized QALYs have been more complicated and therefore more difficult to test empirically. Our comparison will focus first on the assumptions for generalized QALYs, and then on the assumption for (linear) QALYs.

The standard approach to deriving the generalized QALY model is based on three assumptions. First, life duration is utility independent from health quality meaning that preferences between gambles for life duration in a fixed health state are unaffected by the choice of health state. This condition immediately implies SG invariance, but it excludes the reversals of preference due to negative health states. Indeed, one of the advantages of SG invariance is that it is consistent with reversals of strict preference that occur when positive and negative health states are both present in the domain. Second, health quality is utility independent from life duration in the sense that preference between gambles for health quality at a fixed nonzero life duration are unaffected by the choice of life duration. Third, the constant proportional tradeoff condition is adopted: For each health state $Q$ there exists a number $q$ such that $Y$ years in $Q$ is equivalent to $q Y$ years in perfect health, i.e., $(Q, Y)$ is equivalent to (perfect health, $q Y$ ) for all $Y$. The time tradeoff method measures the value of a health quality $Q$ by asking a person to state the number $X$ of years in perfect health that is equivalent to $Y$ years in $Q$. The quotient $X / Y$ is then used as an index of the quality of $Q$. The constant proportional tradeoff condition implies the consistency of this method in the sense that $q=X / Y$ is independent of the particular life duration $Y$. The constant proportional tradeoff condition also implies the zero-condition by substitution of $Y=0$ but it is incompatible with negative health states. Specifically, $Y$ years in a negative health state is not equivalent to any duration in perfect health. Thus, the first and third assumptions can only be applied to positive health states.

The above three conditions plus some regularity conditions imply the special case of the generalized QALY model where all health states are positive and the utility function for life duration is a power function $\left(Y \mapsto Y^{r}\right)$. The result was first derived by Pliskin, Shepard, and Weinstein (1980) which can be considered the first foundation for QALY measurement. We explain here how Theorem 3.1 generalizes and simplifies their result. First note that their preference conditions imply the preference conditions of Theorem 3.1. Therefore by Theorem 
3.1 the Pliskin et al. assumptions imply at least the generalized QALY model. It remains to be shown that, under the assumptions of Pliskin et al., the utility function for life duration is a power utility function. This follows from a standard functional equation that is implied by the constant proportional tradeoff condition $(W(q T)$ $=f(q) \cdot W(T)$ for unknown functions $f, W$; see Aczél 1966, Theorem 3.1.1.4). In this manner, Theorem 3.1 and the functional equation yield Pliskin et al.'s result. Pliskin et al. also demonstrated how adding the assumption of risk neutrality implies the model of Theorem 2.1 for positive health states. This part of their result has already been generalized for positive health states by Bleichrodt et al. (1997) along the lines of Theorem 2.1.

An alternative derivation of the generalized QALY model with exponential utility for life duration instead of power utility was provided by Johannesson, Pliskin, and Weinstein (1994). They assumed that discounted life duration is available as an observable primitive that can be used in axioms, and then extended the traditional preference conditions of linear QALYs to discounted life years. Thus, when the discounting of life years is known a priori, the empirical realism of discounting can be combined with the computational advantages of linear QALYs. In Theorem 3.1, exponential utility for life duration can be obtained by assuming that, for a fixed unequal-to-death health state, a preference between gambles remains unaffected if a constant life duration is added to all life durations involved (Keeney and Raiffa 1976, Theorem 4.15).

The conditions in Theorem 3.1 are more accessible than the commonly adopted assumptions described above. The zero-condition is more transparent and less objectionable than constant proportional tradeoffs, and SG invariance is more elementary than the two utility independence properties. SG invariance has a clear empirical meaning because it refers directly to utility elicitation for life duration by means of standard gambles. The condition is logically weaker than utility independence (and generalized utility independence, see the next section), and thus provides more powerful mathematical results. Nevertheless, it renders the derivations more transparent because SG invariance directly implies linearly related utility functions for life durations. This reasoning suggests that SG invariance captures the essence of generalized QALYs better than the more restrictive conditions that have been used before.

Similarly, the conditions in Theorem 2.1 are simpler and more accessible than the standard assumptions for the (linear) QALY model. It is remarkable and indicative of the force of the zero-condition that of the three standard QALY assumptions, mutual utility independence, constant proportional tradeoffs, and risk neutrality, the first two can simply be dropped.

The advantage of more transparent conditions is that they facilitate the debate on appropriateness of a model. Because the zero-condition is unobjectionable, Theorem 2.1 shows that risk neutrality is the single crucial condition for the QALY model for chronic health states and Theorem 3.1 shows that SG invariance is the single crucial condition for the multiplicative decomposition of the generalized QALY model. As QALYs are widely used but highly debated (Loomes and McKenzie 1989), clarifications of their intrinsic meaning are important. For example, the testing of utility independence of quality from quantity is difficult at a practical level (Miyamoto and Eraker 1988). Our results show that this difficulty is not very central, as this utility independence axiom is implied by the other conditions. Indeed, if the utility independence of quality from duration is violated, Theorems 2.1 and 3.1 show that risk neutrality and SG invariance must also be violated. Therefore, testing utility independence of quality from duration provides an indirect test of risk neutrality and SG invariance.

As a further illustration of the usefulness of Theorem 2.1, assume that the QALY model has been verified for the health states in a specific domain, and now a new health state $Q$ must be incorporated into the analysis. Theorem 2.1 shows that $Q$ can be incorporated into the QALY model as soon as one verifies risk neutrality with respect to $Q$.

We illustrate some examples of phenomena in medical decision analysis that have been raised in debates of QALYs. Figure 5a illustrates an example of "maximum endurable time," first described by Sutherland, Llewelynn-Thomas, Boyd, and Till (1982). The utility functions of the health states $Q_{1}, Q_{2}, Q_{4}, Q_{5}$ satisfy the QALY conditions. This is not so for the utility function for the health state $Q_{3}$. In health state $Q_{3}$, utility increases up to the duration $M$, the maximum endurable 
Figure 5a Utility Functions for Life Duration, an Extreme Example of Maximal Endurable Time $M$ for Health State $Q_{3}$

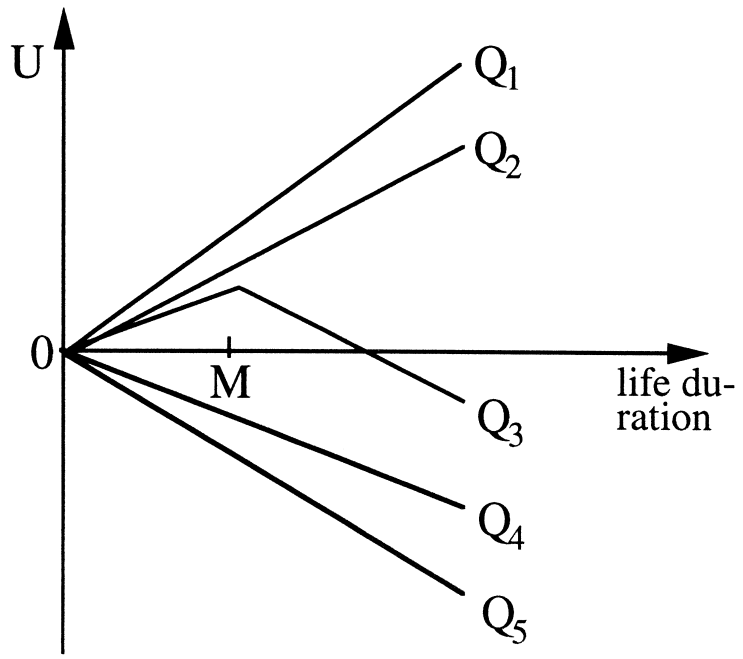

time, after which it decreases. Such a utility function might occur if the individual had a goal to live at least $M$ years, but the accompanying health state $Q_{3}$ was very hard to endure. For example, a patient once related to us that his health state was almost intolerable, but he wanted to live at least five more years in order to see his son graduate from high school. The zero-condition is obviously not violated in Figure 5a, but risk neutrality is violated by the utility in health state $Q_{3}$.

A more realistic case of maximum endurable time is depicted in Figure 5b where utility curves are not straight lines. Here the transition from positive to negative evaluation of life duration for health state $Q_{3}$ is more smooth. The phenomenon of maximum endurable time constitutes a basic counterexample to the generalized QALY model. ${ }^{1}$ Assuming the zero-condition, which is very natural, Theorem 3.1 implies that SG invariance must be violated. Indeed, consider a gamble for life duration where the health state is fixed at $Q_{2}$ and where the certainty equivalent is $M$. If we replace $Q_{2}$ by $Q_{3}$, then $M$ is obviously no longer a certainty equivalent because it is now the unique maximally preferred life duration.

\footnotetext{
${ }^{1}$ Of course, maximum endurable time is a counterexample to the generalized QALY model only if there are other health states whose utility is not extreme at the same value of $M$, e.g., if utility is strictly increasing or decreasing in other health states.
}

Another empirical finding that violates the generalized QALY model (a fortiori, the QALY model as well) is illustrated in Figure 6. Pauker (1976) suggested that people might be unwilling to trade off life duration for improved health quality if life duration were short, but would be willing to make such tradeoffs at longer life durations. Miyamoto and Eraker (1988) observed this phenomenon in a subset of medical patients, and referred to it as indifference to health quality at short durations. Clearly, Figure 6 satisfies the zero-condition, so by Theorem 3.1, SG invariance must be violated. Indeed, the SG probability $p$ to imply that $Y$ is indifferent to a $p$ chance at $X$ and a $1-p$ chance at 0 , is smaller when health is fixed at level $Q_{1}$ (then $p=p_{1}$ ) than when health is fixed at level $Q_{3}$ (then $p=p_{3}$ ).

\section{An Application to General Multiattribute Utility}

This section provides an application of the zerocondition to general multiattribute utility theory. Theorem 3.1 has in fact provided an elementary diagnostic tool for characterizing multiplicative evaluations when 0 points are naturally given that can also be used in general multiattribute utility theory.

To formally develop our result, we assume that the preference domain is the set of simple gambles over a

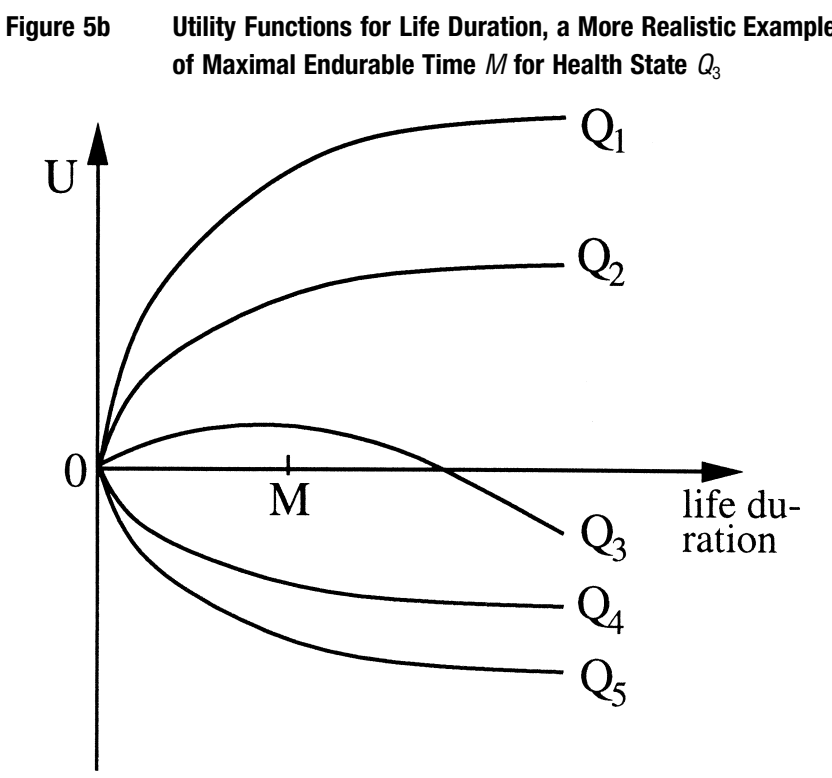


product set $\prod_{j=1}^{n} A_{j}$. Elements of the product set are $n$ tuples $\left(a_{1}, \ldots, a_{n}\right)$. The zero-condition holds for attribute $j$ if there exists a 0 level $z_{j}$ for that attribute, i.e., all $n$ tuples that have $j$ th attribute equal to $z_{j}$ are mutually indifferent. One immediately observes that all $n$-tuples $\left(a_{1}, \ldots, a_{n}\right)$ with one or more attributes at the 0 level are equal in preference, all being equal in preference to an $n$-tuple that takes the 0 level for all attributes for which a 0 level exists.

SG invariance holds for attribute $j$ if an indifference between a two-outcome gamble over attribute $j$ and a certain outcome of attribute $j$, is independent of the level at which the other attributes are kept fixed as long as none of the other attributes is fixed at a 0 level.

THEOREM 5.1. Let $U$ be the von Neumann Morgenstern utility over a product space $A_{1} \times \cdots \times A_{n}$. Assume that there exist 0 levels for attributes $j=1, \ldots, n-1$. Then $S G$ invariance holds for attributes $j=1, \ldots, n-1$ if and only if $U$ is multiplicatively decomposable, i.e., $U\left(x_{1}, \ldots, x_{n}\right)$ $=U_{1}\left(x_{1}\right) \times \cdots \times U_{n}\left(x_{n}\right)$.

Note that the theorem does not assume any structure on the attribute sets $A_{j}$ (other than existence of 0 levels). The theorem shows that we could also have obtained the generalized QALY model by imposing a zerocondition (referring to the health state "dead") and a SG invariance condition on the health state dimension in Theorem 3.1.

The best-known condition in multiattribute utility theory to characterize additive or multiplicative representations is generalized utility independence (Fishburn 1974, Fishburn and Keeney 1974, 1975, von Stengel 1993). It imposes the following two requirements, not only for single attributes, but also for subsets of attributes:

(i) Indifferences are unaffected by levels at which attributes are kept fixed.

(ii) Preferences are either unaffected or reversed if a nonzero level at which attributes are kept fixed is changed into another nonzero level.

The condition implies that the representation is either additive or multiplicative. For the special case of additive representability, a convenient preference condition was introduced by Fishburn (1965), the so-called "marginality" condition, requiring that the value of a gamble over $n$-tuples depend only on the marginal probabilities generated over the single attributes. To the best of our knowledge, no very simple preference axiom exists yet for the characterization of the multiplicative model. Let us also mention that, in general, the restriction of generalized utility independence to single attributes is weaker and only implies a "general multilinear" representation (Keeney and Raiffa 1976).

The above theorem has shown that the implications of the zero-condition are also remarkable in this general context of multiattribute utility. As soon as the zero-condition holds for $n-1$ attributes, the following points hold true:

(1) Generalized utility independence implies the multiplicative model.

(2) The somewhat complicated point (ii) of generalized utility independence can be dropped.

(3) Point (i) of generalized utility independence can be restricted to the case where one gamble has two outcomes, and the other gamble is riskless; that is, the condition can be weakened to SG invariance.

The most remarkable point is:

(4) Generalized utility independence and the weaker SG invariance only need to be imposed on single attributes and not on subsets of attributes. ${ }^{2}$

In the common case where attribute domains are intervals and continuity holds, the zero-condition is automatically satisfied under the multiplicative model if attributes can be subject to sign reversals. Next we discuss some results in general utility theory that are related to Theorem 5.1. The zero-condition is a natural mathematical property that has occurred in several areas. One example is an impressive study of polynomial representations in Krantz, Luce, Suppes, and Tversky (1971, Chapter 7, see the set $S^{0}$ in Definition 7.3). Their model does not consider gambles and invokes more complicated technical conditions than required for multiattribute utility. Another example is Peters (1992, Theorem 11.16), where "simultaneous bargaining games" are studied.

A result, similar to Theorem 3.1 (hence, restricted to the $n=2$ case), appeared in Miyamoto and Eraker (1988, Theorem 1). The main difference between their axioms and ours lies in several technical conditions that

\footnotetext{
${ }^{2}$ Moreover, it need not be imposed on the last attribute in Theorem 5.1 .
} 


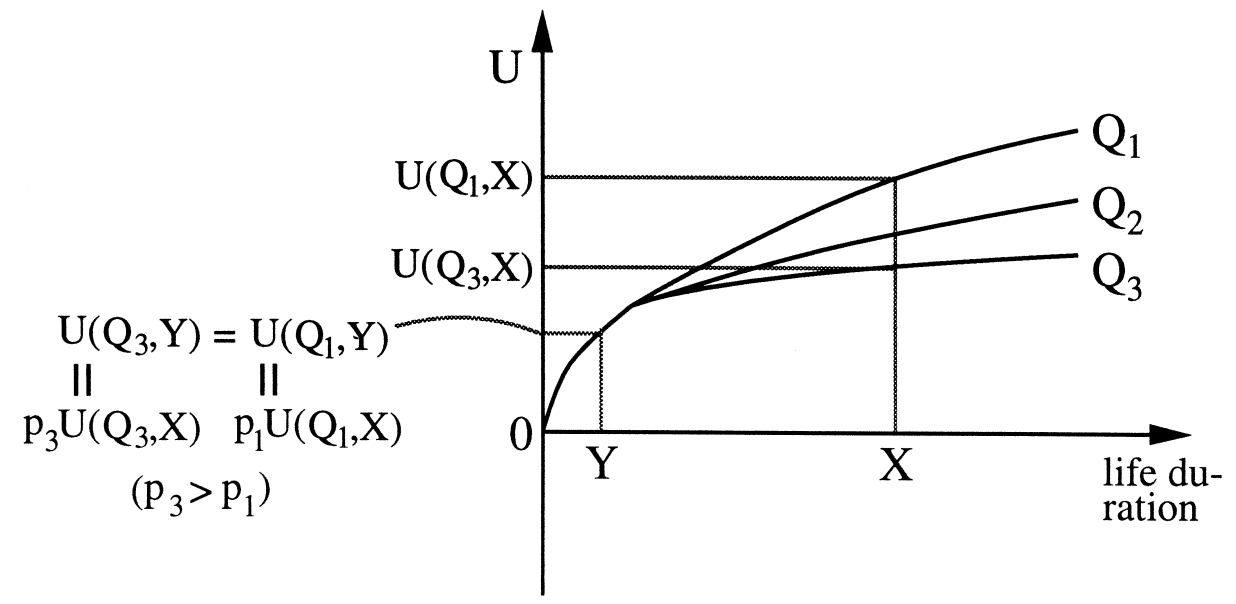

they impose on the utility function. Their Postulate 3 asserts that utility for life duration is continuous, or has at most finitely many discontinuities, and their main theorem assumes that life durations are drawn from a bounded interval. Their Postulate 5 then describes standard gamble invariance for equal-chance gambles (their formulation excludes health states equivalent to death), and their Postulate 4 is the zero-condition. Our Theorem 5.1 generalizes their result by permitting $n>2$, permitting domains that are not intervals, permitting equal-todeath health states, and avoiding continuity assumptions.

A zero-condition for $n=2$ appeared in Fishburn and Rubinstein (1982). They consider two-dimensional objects $(x, t)$ where $x$ designates an amount of money and $t$ the point in time at which $x$ is received. Formally, their first attribute (money) plays a role similar to that of time in our framework, rather than their second attribute (time). Thus, their version of a zero-condition applies to money $(x=0)$ rather than time. The result closest to our theorem is stated informally in their paper after their Lemma 1, where the zero-condition is used implicitly to derive a "Thomsen condition." They assume that money is isomorphic to a real interval, and that time is either discrete or isomorphic to a real interval. They also assume full-force utility independence of money from time, implying that there are no negative or 0 utility values for time. They then show that the zero-condition for money implies a representation $U(x$, $t)=\rho(t)[f(x)-f(0)]+c$. Subtracting the constant $c$ and defining $U_{1}(x)=f(x)-f(0)$ and $U_{2}(t)=\rho(t)$, we see that they have established a multiplicative representation. Fishburn and Rubinstein formulate an explicit multiplicative result in their Theorem 4(iii), but that result is less general than the one just described because further monotonicity and continuity conditions are imposed. They also propose an alternative Condition B3, that either reduces to the classical full-force utility independence condition if there are no negative attributes (in which case it reduces to well-known results of Keeney and Raiffa 1976), or implies the zero-condition in which case it reduces to the above results. Thus our Theorem 5.1 generalizes their result by permitting $n>2$, permitting negative valuations for the second attribute, and avoiding assumptions of interval domains (and continuity).

Next, let us consider the impact of the zero-condition on the propagation of independence conditions from subsets of attributes to their unions and intersections. Fishburn and Keeney (1975), von Stengel (1993), and others demonstrated that, if two overlapping subsets of attributes have certain utility independence properties, then these extend to their union, intersection, differences, etc. These studies did not invoke a zero-condition and thus, utility independence conditions could not be extended from disjoint subsets of attributes to their unions (von Stengel 1993, Counterexample 9). The case is different in the presence of the zero-condition. 
Assume that a subset $A$ of attributes satisfies a SG invariance condition and a zero-condition in the sense that, if its attributes are fixed at some 0 level, then the attributes outside of $A$ do not affect preference. ${ }^{3}$ Assume that the same assumptions hold for a subset $B$ of attributes where $B$ is disjoint from $A$. By Theorem 3.1 (with $A$ in the role of first coordinate and the complementary event $A^{c}$ in the role of second coordinate), the utility function can be decomposed as $U_{A} U_{A^{c}}$. Next we can adopt the zero-condition for $B$ and SG invariance for $B$ to decompose $U_{A^{c}}$ into $U_{B} U_{(A \cup B)^{c}}$. The result is the multiplicative decomposition $U=U_{A} U_{B} U_{(A \cup B)^{c}}$. From this multiplicative decomposition, SG invariance follows for $A \cup B$; also the zero-condition for $A \cup B$ is implied by the zerocondition for $A$ (and also by the zero-condition for $B$ ). In summary, in the presence of the zero-condition, SG invariance extends from $A$ and $B$ to their disjoint union.

One of the main advantages of the zero condition is its simplicity. Indeed, when it is true as in the QALY domain, one can verify its truth in a moment's reflection without an elaborate procedure. The advantage of having such a condition is much like that of Fishburn's (1965) marginality condition for additive utility. In fact, the violation of marginality has been invoked to show that QALY utilities are nonadditive: One would prefer an even chance gamble between (1 year, poor health) and (25 years, excellent health) to an even chance gamble between ( 1 year, excellent health) and (25 years, poor health). The zero condition and marginality provide diagnostics for utility models that can be tested through thought experiments.

\section{Conclusion}

This paper has provided conditions for testing and identifying the appropriateness of the QALY measure for health outcomes. The zero-condition, which is selfevident in health utility applications, leads to a tremendous simplification of existing derivations of QALYs. The zero-condition also leads to substantial simplifications of multiplicative representations in general multiattribute utility theory.

\footnotetext{
${ }^{3}$ This condition is implied by a zero-condition for any attribute within A.
}

Appendix

\section{Proofs}

ProOf OF THEOREM 2.1. Necessity of the conditions is obvious. For sufficiency, first note that risk neutrality implies that for every health state $Q$ the utility function for life duration is linear, so is of the form $V(Q) \cdot T+A(Q)$, where $V(Q)$ and $A(Q)$ depend on $Q$ but are independent of $T$; this is illustrated in Figure 2a. The zero-condition implies that $U(Q, 0)=U\left(Q^{\prime}, 0\right)$, hence $A(Q)=A\left(Q^{\prime}\right)$ for all $Q$ and $Q^{\prime}$. It is well known that one is free to subtract a constant from a von Neumann Morgenstern utility function, hence let us redefine $U$ such that $A(Q)$ $=0$ for all $Q$. (Note here that the constant that one subtracts should be the same for all $(Q, T)$ so in particular should be independent of Q.) The QALY model $U(Q, T)=V(Q) \cdot T$ follows immediately.

PROOF OF THEOREM 3.1. Necessity of the preference conditions for the generalized QALY model follows from substitution. Hence we assume the preference conditions and derive the generalized QALY model. All equal-to-death chronic health states are equivalent, and we may assume that utility is zero at those. Setting $V(Q)=0$ provides the generalized QALY formula for these. Next we consider unequal-todeath health states $Q$. Fix any such health state $H$ (e.g., perfect health), and write $W(T)=U(H, T)$ for all life durations $T$. For any unequalto-death health state $Q$, all standard gamble equivalences between $(Q$, $\left.T^{\prime}\right)$ and gambles yielding $(Q, T)$ with probability $p$ and $(Q, S)$ with probability $1-p$ are the same as for $H$. Hence there exist numbers $V(Q)$ and $A(Q)$ so that $U(Q, T)=V(Q) W(T)+A(Q)$, where $V(Q)$ can be positive or negative. $A(Q)=0$ follows as in the proof of Theorem 2.1 (it is also imposed here by the convention that $U$ be 0 at equal-to-death health states). The generalized QALY model follows.

Remark A1. The result of Theorem 3.1 is also mathematically correct if we permit values $T<0$. It does not invoke any structure on the set of time points (or health states) other than existence of a $T=0$ level. That was the only assumption used in the proof.

Proof OF THEOREM 5.1. Again, SG invariance easily follows from the representation. So we assume SG invariance for attributes $j=1$, $\ldots, n-1$ and derive the representation.

It was explained in the text that all $n$-tuples $\left(a_{1}, \ldots, a_{n}\right)$ for which at least one attribute is at the 0 level are equal in preference. Hence we can set the von Neumann Morgenstern utility function $U$ equal to 0 whenever any attribute attains a 0 level, and that is what we will assume henceforth.

First consider the product set $A_{1} \times \cdots \times A_{n}$ as a two-fold product $A_{1} \times\left(A_{2} \times \cdots \times A_{n}\right)$. To this two-fold product set we will apply Theorem 3.1. As the zero-condition also holds for attribute 1 in this two-fold product, only the SG invariance condition remains to be verified. Assume that the "second coordinate" $\left(a_{2}, \ldots, a_{n}\right)$ is fixed at a level that is nonzero in the sense of the two-fold product, i.e., preference is affected by the first coordinate $a_{1}$ in $\left(a_{1}, a_{2}, \ldots, a_{n}\right)$. Then obviously none of the values $a_{2}, \ldots, a_{n}$ can be a 0 level in the $n$ dimensional product $A_{1} \times \cdots \times A_{n}$. Therefore SG invariance in the $n$ dimensional product $A_{1} \times \cdots \times A_{n}$ implies SG invariance in the two-fold product $A_{1} \times\left(A_{2} \times \cdots \times A_{n}\right)$. We can now invoke Theorem 3.1 and Remark A1 and conclude that 


$$
U\left(a_{1}, a_{2}, \ldots, a_{n}\right)=U_{1}\left(a_{1}\right) \cdot W\left(a_{2}, \ldots, a_{n}\right) .
$$

Note that in the proof of Theorem 3.1, a constant was subtracted from the utility function $U$ to obtain the multiplicative form. Here, however, such a constant need not be subtracted anymore because we have already set $U$ equal to 0 when attributes take their 0 level. This comment also applies to subsequent steps in the proof, where again Theorem 3.1 will be invoked but no constant needs to be added to or subtracted from $U$.

The proof of the theorem now proceeds by fixing $a_{1}$ at any nonzero level, say $b_{1}$, and studying the preference relation over the $n-1$ dimensional space $\left\{b_{1}\right\} \times A_{2} \times \cdots \times A_{n}$. We may assume that $U_{1}\left(b_{1}\right)$ $>0$, otherwise we replace $U_{1}$ by $-U_{1}$ and $W$ by $-W$. On the $n-1$ dimensional space, expected utility holds with $W\left(a_{2}, \ldots, a_{n}\right)$ as utility function. Here we can again apply Theorem 3.1, as was done before (now consider the two-dimensional product $A_{2} \times\left(\left\{b_{1}\right\} \times A_{3} \times \cdots \times\right.$ $\left.\left.A_{n}\right)\right)$, to obtain

$$
W\left(a_{2}, a_{3}, \ldots, a_{n}\right)=U_{2}\left(a_{2}\right) \times W^{*}\left(a_{3}, \ldots, a_{n}\right) .
$$

Thus we can proceed, after $n-1$ steps the decomposition $U\left(a_{1}, \ldots\right.$, $\left.a_{n}\right)=U_{1}\left(a_{1}\right) \cdot U_{2}\left(a_{2}\right) \cdot \cdots U_{n-1}\left(a_{n-1}\right) \cdot W^{* *}\left(a_{n}\right)$ results, and we write $U_{n}$ $=W^{* *}$ to obtain the multiplicative representation in the theorem.

\section{References}

Aczél, J., "Lectures on Functional Equations and Their Applications," Academic Press, New York, 1966.

Bleichrodt, H., P. P. Wakker, and M. Johannesson, “Characterizing QALYs by Means of Risk Neutrality," J. Risk and Uncertainty, 15 (1997), 107114.

Clemen, R., "Making Hard Decisions: An Introduction to Decision Analysis," PWS-Kent, Boston, MA, 1991.

Currim, I. S. and R. K. Sarin, "Prospect Versus Utility," Management Science, 35 (1989), 22-41.

de Finetti, B., "La Prévision: Ses Lois Logiques, ses Sources Subjectives," Annales de l'Institut Henri Poincaré, 7 (1937), 1-68. Translated into English by H. E. Kyburg, "Foresight: Its Logical Laws, its Subjective Sources," in H. E. Kyburg and H. E. Smokler (Eds.), Studies in Subjective Probability, Wiley, New York, 1964, 53-118; 2nd ed., Krieger, New York, 1980.

Fishburn, P. C., "Independence in Utility Theory with Whole Product Sets," Oper. Res., 13 (1965), 28-45.

-, "Von Neumann-Morgenstern Utility Functions on Two Attributes," Oper. Res., 22 (1974), 35-45.

— and R. L. Keeney, "Seven Independence Concepts and Continuous Multiattribute Utility Functions," J. Math. Psychology, 11 (1974), 294-327.

— and _ "Generalized Utility Independence and Some Implications," Oper. Res., 23 (1975), 928-940.
— and G. A. Kochenberger, "Two-Piece von Neumann-Morgenstern Utility Functions," Decision Sciences, 10 (1979), 503-518.

- and A. Rubinstein, "Time Preference," International Economic Review, 23 (1982), 677-694.

Johannesson, M., "The Ranking Properties of Healthy-Years Equivalents and Quality-Adjusted Life-Years under Certainty and Uncertainty," International J. Technology Assessment in Health Care 11 (1995), 40-48.

- J. S. Pliskin, and M. C. Weinstein, "A Note on QALYs, Time Tradeoff, and Discounting," Medical Decision Making, 14 (1994), 188-193.

Keeney, R. L. and H. Raiffa, “Decisions with Multiple Objectives,, Wiley, New York, 1976; 2nd ed., Cambridge University Press; Cambridge, England, 1993.

Krantz, D. H., R. D. Luce, P. Suppes, and A. Tversky, "Foundations of Measurement, Vol.I. (Additive and Polynomial Representations)," Academic Press, New York, 1971.

Loomes, G. and L. McKenzie, "The Use of QALYs in Health Care Decision Making," Social Science and Medicine, 28 (1989), 299-308.

Miyamoto, J. M., and S. A. Eraker, "A Multiplicative Model of the Utility of Survival Duration and Health Quality," J. Experimental Psychology, General 117 (1988), 3-20.

Nau, R. F., 'Indeterminate Probabilities on Finite Sets," Ann. Statistics, 20 (1992), 1737-1767.

Pauker, S. G., "Coronary Artery Surgery: The Use of Decision Analysis," Ann. Internal Medicine, 85 (1976), 8-18.

Peters, H. J. M., "Axiomatic Bargaining Theory," Kluwer Academic Publishers, Dordrecht, 1992.

Pliskin, J. S., D. S. Shepard, and M. C. Weinstein, “Utility Functions for Life Years and Health Status," Oper. Res., 28 (1980), 206-224.

Sox, H. C., M. A. Blatt, M. C. Higgins, and K. I. Marton, "Medical Decision Making," Buttersworths, Boston, MA, 1986.

Sutherland, H. J., R. N. J. Llewelynn-Thomas, N. F. Boyd, and J. E. Till, "Attitudes Toward Quality of Life: The Concept of 'Maximal Endurable Time'," Medical Decision Making, 2 (1982), 299-309.

Tversky, A. and D. Kahneman, "Advances in Prospect Theory: Cumulative Representation of Uncertainty," J. Risk and Uncertainty, 5 (1992), 297-323.

von Neumann, J. and O. Morgenstern, "Theory of Games and Economic Behavior," Princeton University Press, Princeton, NJ, 1944, 1947, 1953.

von Stengel, B., "Closure Properties of Independence Concepts for Continuous Utilities," Math. Oper. Res., 18 (1993), 346389.

Weinstein, M. C., H. V. Fineberg, A. S. Elstein, H. S. Frazier, D. Neuhauser, R. R. Neutra, and B. J. McNeil, “Clinical Decision Analysis," Saunders, Philadelphia, PA, 1980.

Accepted by Robert Nau; received July 25, 1996. This paper has been with the authors 2 months for 1 revision. 